\title{
HLA-A*2402-Restricted URLC10-TTK-KOC1 Multipeptide Vaccine
}

National Cancer Institute

\section{Source}

National Cancer Institute. HLA-A*2402-Restricted URLC10-TTK-KOC1 Multipeptide

Vaccine. NCI Thesaurus. Code C77897.

A cancer vaccine containing three HLA-A*2402-restricted peptide epitopes with potential immunostimulatory and antitumor activities. Peptide epitopes in this vaccine are derived from URLC10 (up-regulated lung cancer 10); TTK (TTK protein kinase); and KOC1 (IGF II mRNA Binding Protein 3). Upon administration, URLC10-TTK-KOC1 multipeptide vaccine may stimulate a cytotoxic T lymphocyte $(\mathrm{CT} L)$ response against tumor cells expressing URLC10, TTK and KOC1 peptides, resulting in tumor cell lysis and decreased tumor growth. HLA-A*2402 is an MHC class I molecule that presents antigenic peptides to CD8+ T cells; epitope design restricted to epitopes that bind most efficiently to HLAA*2402 may improve antigenic peptide immunogenicity. 